\title{
ON THE MIXED INTEGER RANDOMIZED PATTERN SEARCH ALGORITHM
}

\author{
EBERT BREA
}

\begin{abstract}
We analyze the convergence and performance of a novel direct search algorithm for identifying at least a local minimum of unconstrained mixed integer nonlinear optimization problems. The Mixed Integer Randomized Pattern Search Algorithm (MIRPSA), so-called by the author, is based on a randomized pattern search, which is modified by two main operations for finding at least a local minimum of our problem, namely: moving operation and shrinking operation. The convergence properties of the MIRPSA are here analyzed from a Markov chain viewpoint, which is represented by an infinite countable set of states $\{d(q)\}_{q=0}^{\infty}$, where each state $d(q)$ is defined by a measure of the $q$ th randomized pattern search $\mathcal{H}_{q}$, for all $q \in \mathbb{N}$. According to the algorithm, when a moving operation is carried out on a $q$ th randomized pattern search $\mathcal{H}_{q}$, the MIRPSA Markov chain holds its state. Meanwhile, if the MIRPSA carries out a shrinking operation on a $q$ th randomized pattern search $\mathcal{H}_{q}$, the algorithm will then visit the next $(q+1)$ th state. Since the MIRPSA Markov chain never goes back to any visited state, we therefore say that the MIRPSA yields a birth and miscarriage Markov chain.
\end{abstract}

\section{INTRODUCTION}

Consider the following unconstrained mixed integer nonlinear problem:

$$
\underset{(x, y) \in \mathbb{R}^{n} \times \mathbb{Z}^{m}}{\operatorname{minimize}} f(x, y),
$$

where $f(x, y): \mathbb{R}^{n} \times \mathbb{Z}^{m} \rightarrow \mathbb{R}$ is a nonlinear objective function, for which an analytical and explicit mathematical expression cannot be obtained. In this case, the objective function must be evaluated by an appropriate simulation model, and $x \in \mathbb{R}^{n}$ and $y \in \mathbb{Z}^{m}$ are decision variables of our problem. Of course, there exists no derivative function due to the objective function being defined in the set $\mathbb{R}^{n} \times \mathbb{Z}^{m}$.

This class of problems often arises out of practical applications, which are frequently studied in the engineering design field, wherein there exist both real and discrete variables. Examples of these problems are shown by Floudas [3], who presents a wide set of chemical engineering problems. Moreover, mixed integer

Key words and phrases. Mixed integer nonlinear optimization; Direct search optimization.

The author would like to thank the Council for Scientific and Humanistic Development of the Universidad Central de Venezuela for financially supporting this research. 
optimization problems are also applied in the design of electrical motors. For example, Brea [2] presents an application of this problem for optimal design of a permanent magnet electric machine.

For identifying at least a local minimum of the problem given by Eq. (1.1), we have considered the use of randomized pattern search in the Euclidean space $\mathbb{R}^{n} \times$ $\mathbb{Z}^{m}$. The idea of using a randomized pattern search was put forward by Lawrence and Fawzi [8, 7], who proposed an algorithmic method of linear and nonlinear optimization problems when the set of decision variables are real. Nevertheless, the authors do not analyze any convergence property of their algorithm.

There exists an abundant literature about the use of deterministic pattern search for identifying optimum solution in nonlinear optimization problems; for instance, Kolda and coworkers [5, 6], and also Torczon [11] do a deep study on convergence of pattern search algorithms. In addition, Hart 4 presents a convergence analysis of evolutionary pattern search, wherein he analyzes a class of evolutionary algorithm, based on pattern search algorithm, for unconstrained and bound constrained optimization problems. Audet and Dennis [1] also propose a pattern search algorithm for mixed integer optimization problems.

Nevertheless, the author here proposes a novel randomized pattern search for finding solutions to mixed integer unconstrained optimization problems. The Mixed Integer Randomized Pattern Search Algorithm (MIRPSA), so-called by the author, is based on a mixed integer randomized pattern search, which is modified by the MIRPSA for identifying at least a local minimum of our nonlinear optimization problems.

The MIRPSA is easy to code; however, studying the properties of its convergence is not so straightforward. Moreover, this can be considered a great challenge. We study its convergence properties using a Markov chain approach, where each state $d(q)$ for all $q \in \mathbb{N}$ represents a measure of the randomized pattern search $\mathcal{H}_{k}$ at each $k$ th iteration of the algorithm. Therefore, the Markov chain is an infinite countable set of state space $\Lambda=\{d(q)\}_{q=0}^{\infty}$.

Our goal is focused on convergence properties of the MIRPSA using a Markov chain viewpoint, because of the fact that each $q$ th state is given by a measure of the randomized pattern search, and allows us to estimate the probability of being at each $q$ th state.

The MIRPSA starts with an initial guess 0th mixed integer randomized pattern search $\mathcal{H}_{0}$, which is used for locating an initial set of randomized trial points, in order to evaluate the objective function at each trial point for comparing with the center of the randomized pattern search function value $f\left(c_{k}\right)$. Depending on these comparisons with the center of the randomized pattern search function value $f\left(c_{k}\right)$, can be executed a moving operation or shrinking operation on the current mixed integer randomized pattern search $\mathcal{H}_{0}$. Namely the MIRPSA is basically based on two main operations: moving operation and shrinking operation, on any $k$ th mixed integer randomized pattern search $\mathcal{H}_{k}$, which is used for locating a set of randomized trial points at each $k$ th iteration around the center of the randomized pattern search $c_{k}$. The pattern search center will be moved to the best trial point, 
if the objective function value at the best trial point is better than the objective function value at the current center $c_{k}$. In contrast, the pattern search center will remain unchanged, but pattern search range will shrink for both real and integer factors, if the objective function value at the best trial point does not improve the objective function at the center of the current pattern search. This procedure is iteratively repeated until some stopping rule is satisfied.

The remainder of the paper is arranged as follows. A general description, together with a pseudocode of the MIRPSA, are provided in Section 2. In Section 3 we describe a general Markov chain framework that is produced when the MIRPSA is being carried out in order to analyze its convergence properties. A low dimension case is studied in Section 4 and a simple numerical example is also reported. In Section 5 we show the performance of the MIRPSA when the objective function is a multidimensional quadratic function, and we also report results of some numerical test problems. Finally, conclusions and suggestions for future research are provided in Section 6.

\section{The MiXed INTEGER RANDOMIZED PATTERN SEARCH ALGORITHM}

The MIRPSA, displayed in Fig. 1, is based on a randomized pattern search $\mathcal{H}_{k}$, which is defined by a set of random trial points at each $k$ th iteration for identifying at least a local optimum point of the problem presented by Eq. (1.1). Each random trial point $z \in \mathbb{R}^{n} \times \mathbb{Z}^{m}$ is generated by random locations around each $k$ th pattern search center $c_{k}=\left(c^{(1)}, \ldots, c^{(n)}, c^{(n+1)}, \ldots, c^{(n+m)}\right)^{t} \in \mathbb{R}^{n} \times \mathbb{Z}^{m}$ using continuous and discrete uniform distributions. Each $i$ th real component of each trial point is randomly generated by

$$
z^{(i)} \leftarrow c^{(i)}+\mathrm{u}\left(-\delta^{[k]}, \delta^{[k]}\right), \quad \forall i=1, \ldots, n,
$$

meanwhile their integer components are yielded by

$$
z^{(i)} \leftarrow c^{(i)}+\mathrm{u}\left(-\max \left(1, \Delta^{[k]}\right), \max \left(1, \Delta^{[k]}\right)\right), \quad \forall i=n+1, \ldots, n+m,
$$

where $\delta^{[k]}$ and $\Delta^{[k]}$ denote, respectively, the parameters of continuous and discrete uniform distribution at each pattern search $\mathcal{H}_{k}$.

The MIRPSA has two main operations: moving operation and shrinking operation, of which only one can be carried out on the random pattern search at each $k$ th iteration. A moving operation is executed at the $k$ th iteration, when a trial point $z$ at least improves the current value objective function $f\left(v_{k}\right)$. That is, if $f(z)<f\left(v_{k}\right)$, then the center of the current randomized pattern search will be moved to the best trial point of the set of $M$ random trial points yielded by the MIRPSA at each $k$ th iteration. Meanwhile a shrinking operation will be carried out at the $k$ th iteration, if the current pattern search center is better than all trial points at the current $k$ th iteration. In this last case, the size of the randomized pattern search is shrunk by the algorithm at a rate of $\alpha$ in order to reduce the range of a continuous uniform distribution, and $\beta$ for shrinking the step-length of a discrete uniform distribution. 


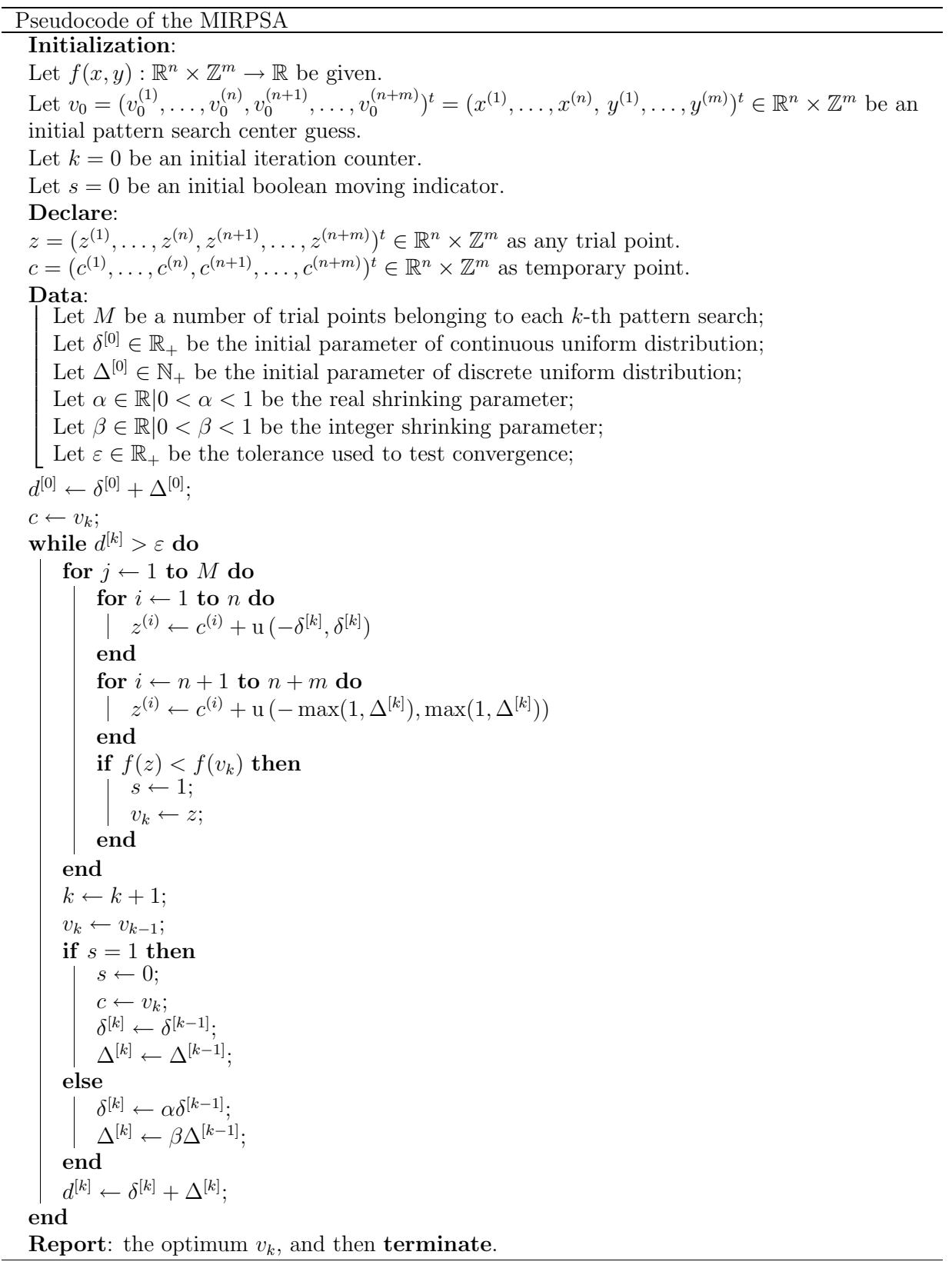

Figure 1. Mixed Integer Randomized Pattern Search Algorithm

These operations are performed until the stopping criterion of the MIRPSA is met, in our case, when $\alpha \delta^{[k-1]}+\beta \Delta^{[k-1]} \leq \varepsilon$, where $\varepsilon>0$ is the tolerance for testing the stopping criterion rule. 


\section{On the CONVERGENCE OF THE MIRPSA}

In this section, we analyze the convergence of the MIRPSA from a Markov chain viewpoint, wherein the set of discrete state space is a countable and infinite Markov chain $\{d(q)\}_{q=0}^{\infty}$, where $d(q)$ is given by a size measure of each $k$ th random pattern search $\mathcal{H}_{k}$ at each $k$ th iteration of the MIRPSA for each $q$ th state. The main convergence property is based on the fact that the MIRPSA visits each $q$ th state of the Markov chain $\{d(q)\}_{q=0}^{\infty}$, when the MIRPSA is applied to a convex minimization problem.

Consider the MIRPSA as a Markov chain, where the set of state space is defined by $\{d(q)\}_{q=0}^{\infty}$. Here $d(q)$, for each $q \in \mathbb{N}$, represents a measure of the pattern search size of $\mathcal{H}_{k}$ at each $k$ th iteration, which holds on a $q$ th state within a measurable set of $N_{q}$ successive moving operations that have been carried out at each $k$ th iteration.

Thus, for each $k \in \mathbb{N}, d(k)=\delta^{[k]}+\Delta^{[k]}$, and this is computed by $d(k)=$ $\alpha \delta^{[k-1]}+\beta \Delta^{[k-1]}$. Notice that $d(k)=d(0)$, for all $k \in\left\{\hat{k} \in \mathbb{N}: 0 \leq \hat{k}<N_{0}\right\}$; $d(k)=d(q)$, for all $k \in\left\{\hat{k} \in \mathbb{N}: \sum_{i=0}^{q-1} N_{i} \leq \hat{k}<\sum_{i=0}^{q} N_{i}\right\}$; and $d(q)>d(q+1)$, for all $q \in \mathbb{N}$, where $N_{i}$ is the amount of successive moving operations that are carried out by the MIRPSA at each $i$ th state.

Fig. 2 depicts the Markov chain of the MIRPSA, where, without loss of generality, each $d(q)$ state is denoted by its $q$ th number in order to simplify the notation in the figure. We can say that this Markov chain represents a birth and miscarriage discrete process, because at any $k$ th iteration, the process may go from each $q$ th state to the next $(q+1)$ th state, so called birth, or it may remain at its $q$ th state, what would be considered a miscarriage. Notice that the MIRPSA never goes back from any $q$ th state to any visited state $\{d(q)\}_{q=0}^{q-1}$. The MIRPSA holds on the same $q$ th state due to a set of $k$ successive moving operations, for all $\sum_{i=0}^{q} N_{i} \leq k<\sum_{i=0}^{q+1} N_{i}$ iterations, where $N_{i}$ is the total number of iterations, wherein the MIRPSA remains at an $i$ th state. Meanwhile, it changes from a $q$ th state into the following $(q+1)$ th state because of a shrinking operation, which takes place at each $\left(\sum_{i=0}^{q} N_{i}\right)$ th iteration.

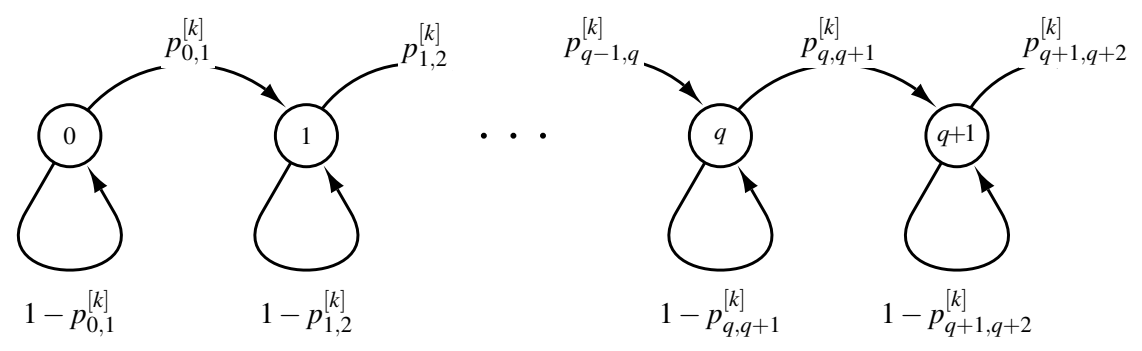

FIGURE 2. Markov chain graph of the MIRPSA 
We can assert that for all

$$
k \in\left\{\hat{k} \in \mathbb{N}: \sum_{i=0}^{q} N_{i} \leq \hat{k}<\sum_{i=0}^{q+1} N_{i}\right\}
$$

and $q \in \mathbb{N}$, the probability that any moving operation has taken place is given by

$$
\mathcal{P}\left\{D_{k+1}=d(q) \mid D_{k}=d(q)\right\}=\mathcal{P}_{k}(d(q), d(q))=1-p_{q, q+1}^{[k]} .
$$

On the other hand, we also say that for each $k=\sum_{i=0}^{q} N_{i}$ and $q \in \mathbb{N}$, the probability that a shrinking operation has been carried out at each $k$ th iteration is

$$
\mathcal{P}\left\{D_{k+1}=d(q+1) \mid D_{k}=d(q)\right\}=\mathcal{P}_{k}(d(q), d(q+1))=p_{q, q+1}^{[k]} .
$$

We denote the initial distribution by

$$
\pi_{0}(d(0))=\mathcal{P}\left\{D_{0}=d(0)\right\},
$$

where the subscript 0 of $\pi_{0}(d(0))$ means the 0th iteration, and the argument represents the state $d(0)$. by

We similarly say that at each $k$ th iteration, the $q$ th state distribution is denoted

$$
\pi_{k}(d(q))=\mathcal{P}\left\{D_{k}=d(q)\right\} .
$$

3.1. Estimating the probability of miscarriage. We will show that the MIRPSA obtains $h$ trial points from $M$ samples of the pattern search $\mathcal{H}_{q}$ with binomial distribution.

Proposition 3.1. Assume that a measure of size of any kth mixed integer randomized pattern search $\mathcal{H}_{k} \in \mathbb{R}^{n} \times \mathbb{Z}^{m}$ is given by $\alpha^{k} \delta^{[0]}+\beta^{k} \Delta^{[0]}$. If the stochastic process $\{d(q)\}_{q=0}^{\infty}$, whose state is given by $d(0)=\delta^{[0]}+\Delta^{[0]} ; d(k)=d(0), \forall k \in\{\hat{k} \in$ $\left.\mathbb{N}: 0 \leq \hat{k}<N_{0}\right\} ;$ or $d(k)=d(q), \forall k \in\left\{\hat{k} \in \mathbb{N}: \sum_{i=0}^{q-1} N_{i} \leq \hat{k}<\sum_{i=0}^{q} N_{i}\right\}$, where $N_{i}$ is the amount of successive moving operations carried out by the MIRPSA at each qth state, or $d(k+1)=\alpha \delta^{[k]}+\beta \Delta^{[k]}$ for all $k \in \mathbb{N}$, then the MIRPSA is a non homogeneous Markov chain.

Proof. The MIRPSA goes from $d(q)$ to $d(q+1)$ due to a shrinking operation, wherein the parameters of both continuous and discrete uniform distributions are respectively updated by $\delta^{[q+1]}=\alpha \delta^{[q]}$ and $\Delta^{[q+1]}=\beta \Delta^{[q]}$, what causes a new uniform distribution at the $q$ th state. Finally, since only one shrinking operation is carried out by the algorithm at each $q$ th state, and only sampling trial of the current mixed integer randomized pattern search $\mathcal{H}_{k} \in \mathbb{R}^{n} \times \mathbb{Z}^{m}$ are used to determine where to move next, $\{d(q)\}_{q=0}^{\infty}$ has the memoryless property, what causes that the MIRPSA just goes from a $d(q)$ to $d(q+1)$ for all $q \in \mathbb{N}$, and this fact allows us to ensure that the stochastic process is a non homogeneous Markov chain.

Remark 3.2. Note that each $k$ th mixed integer randomized pattern search $\mathcal{H}_{k} \in$ $\mathbb{R}^{n} \times \mathbb{Z}^{m}$ is contained in a convex hull $C\left(\mathcal{H}_{k}\right)$, and its $(n+m)$ dimensional volume for each qth state is given by

$$
v(q)=2^{n+m}\left(\delta^{[q]}\right)^{n}\left(\max \left(1, \Delta^{[q]}\right)\right)^{m}, \quad \forall q \in \mathbb{N} .
$$


Proposition 3.3. Assume that the MIRPSA carries out $k \geq 0$ iterations, and also that each qth state is non absorbing. If the MIRPSA stops its iterations when the stopping rule given by $\alpha \delta^{[q-1]}+\beta \Delta^{[q-1]} \leq \varepsilon$ is held, and $\varepsilon>0$, then the MIRPSA visits a finite number $Q$ of states given by

$$
Q=\inf \left\{\widehat{Q} \in \mathbb{N}: \alpha^{\widehat{Q}} \delta^{[0]}+\beta^{\widehat{Q}} \Delta^{[0]} \leq \varepsilon\right\}
$$

Proof. Since the MIRPSA updates at each $q$ th state because a shrinking operation is carried out, then $d(q)=\alpha^{M} \delta^{[0]}+\beta^{M} \Delta^{[0]}$, where $M$ is the number of shrinking operations until the stopping rule is held, $\delta^{[0]}$ and $\Delta^{[0]}$ are, respectively, the initial parameters sampling continuous and discrete uniform distributions, and we have assumed that each $q$ th visited state is non absorbing. Hence, the number of visited states by the MIRPSA is given by Eq. [3.5).

Of course, the previous proposition needs proving the assumption that each $q$ th state is non absorbing, which will be proved later, in Lemma 3.6.

Proposition 3.4. Let $z_{j}^{[k]} \in \mathbb{R}^{n} \times \mathbb{Z}^{m}$ be the $j$ th trial point of the kth mixed integer randomized pattern search $\mathcal{H}_{k}$, and let $\nu_{q}$ denote the maximum number of iteration wherein the MIRPSA is at the qth state. Assume $M$ sampling of trial points of a kth randomized pattern search $\mathcal{H}_{k}$, which are independent Bernoulli trials with probability $\rho_{q, k+\nu}=\mathcal{P}\left\{\tilde{f}\left(z_{j}^{[q, k+\nu]}\right)<f\left(c^{[q, k+\nu]}\right)\right\}$, where $k$ is the iteration counter, wherein the MIRPSA arrived at the qth state, $\nu \in\left[0, \nu_{q}\right]$ is number of iteration carried out by the MIRPSA at each qth state, that is, for the same pattern search $\mathcal{H}_{q}$, and $\tilde{f}\left(z_{j}^{[q, k+\nu]}\right)$ denotes the random value of each jth random trial point, which is obtained by evaluation of the objective function at each jth trial point for each $k$ th iteration; and $f\left(c^{[q, k+\nu]}\right)$ is the deterministic value of the objective function at the $k$ th randomized pattern search center point.

Then the probability that a moving operation takes place at each $(k+\nu)$ th iteration of a qth state is given by

$$
\mathcal{P}_{k+\nu}(d(q), d(q))=p_{q, q}^{[k+\nu]}=\sum_{h=1}^{M}\left(\begin{array}{c}
M \\
h
\end{array}\right) \rho_{q, k+\nu}^{h}\left(1-\rho_{q, k+\nu}\right)^{M-h} .
$$

Proof. Due to the fact that the MIRPSA takes $M$ independent Bernoulli trial points on the $q$ th randomized pattern search $\mathcal{H}_{q}$, the number $h$ of all the $j$ th trial points whose $\tilde{f}\left(z_{j}^{[q, k+\nu]}\right)<f\left(c^{[q, k+\nu]}\right)$ is given by a binomial distribution $B\left(M, \rho_{q, k+\nu}\right)$.

Hence, from Proposition 3.4, we can also assert

$$
\mathcal{P}_{k+\nu}(d(q), d(q+1))=p_{q, q+1}^{[k+\nu]}=\left(1-\rho_{q, k+\nu}\right)^{M} .
$$

As we can see each $q$ th center point $c^{[q, k+\nu]}$ is known from the best trial point at the $(k+\nu-1)$ th iteration for each $\nu$.

Notice that for each $(k+\nu)$ th iteration and for each $j$ th random trial point

$$
\mathcal{P}\left\{\tilde{f}\left(z_{j}^{[q, k+\nu]}\right)<f\left(c^{[q, k+\nu]}\right)\right\}+\mathcal{P}\left\{\tilde{f}\left(z_{j}^{[q, k+\nu]}\right) \geq f\left(c^{[q, k+\nu]}\right)\right\}=1 .
$$


It is worthwhile pointing out that $\rho_{q, k+\nu}=\mathcal{P}\left\{\tilde{f}\left(z_{j}^{[q, k+\nu]}\right)<f\left(c^{[q, k+\nu]}\right)\right\}$; this mainly depends on the dimension of the problem and pattern search size.

We now turn to the estimation of the probability of miscarriage at each $d(q)$ state of the MIRPSA.

Theorem 3.5. Let $\nu$ be the quantity of iterations that has been carried out by the MIRPSA at any qth state $d(q)$, for denoting the qth state without affecting the generality. The probability that then occur $\nu$ iterations at the qth state of the MIRPSA (this means $\nu$ moving operations after $k$ iteration of the MIRPSA), is given for each $k \in \mathbb{N}, \nu \in \mathbb{N}_{+}, q \in \mathbb{N}_{+}$by

$$
\begin{aligned}
\mathcal{P}\left\{D_{k+\nu}=d(q)\right\}= & \sum_{n=0}^{\nu-1} p_{q-1, q}^{[k+n]} \prod_{m=n+1}^{\nu-1}\left(1-p_{q, q}^{[k+m]}-p_{q-1, q}^{[k+m]}\right) \\
& +\prod_{m=0}^{\nu-1}\left(1-p_{q, q}^{[k+m]}-p_{q-1, q}^{[k+m]}\right) \mathcal{P}\left\{D_{k}=d(q)\right\},
\end{aligned}
$$

where, in general, $p_{q, q}^{[\cdot]}$ is the probability that a moving operation occurs, that is, the probability of keeping the qth state at [.] iteration; $p_{q-1, q}^{[\cdot]}$ is the probability that a shrinking operation has taken place at [.] iteration.

Proof. Since

$$
\mathcal{P}\left\{D_{1}=d(0)\right\}=\mathcal{P}\left\{D_{1}=d(0), D_{0}=d(0)\right\}
$$

we easily get

$$
\mathcal{P}\left\{D_{1}=d(0)\right\}=\mathcal{P}\left\{D_{1}=d(0) \mid D_{0}=d(0)\right\} \mathcal{P}\left\{D_{0}=d(0)\right\},
$$

what allows us to assert, according to Eq. (3.1),

$$
\mathcal{P}\left\{D_{1}=d(0)\right\}=\left(1-p_{0,0}^{[0]}\right) \mathcal{P}\left\{D_{0}=d(0)\right\},
$$

where $\mathcal{P}\left\{D_{0}=d(0)\right\}=\pi_{0}(d(0))$ is given by Eq. (3.3), for all $k=0,1, \ldots, N_{0}-1$;

$$
\begin{aligned}
\mathcal{P}\left\{D_{0}=d(0)\right\} & =\pi_{0}(d(0)) ; \\
\mathcal{P}\left\{D_{1}=d(0)\right\} & =\left(1-p_{0,0}^{[0]}\right) \mathcal{P}\left\{D_{0}=d(0)\right\} ; \\
\mathcal{P}\left\{D_{2}=d(0)\right\} & =\left(1-p_{0,0}^{[1]}\right) \mathcal{P}\left\{D_{1}=d(0)\right\} ; \\
\mathcal{P}\left\{D_{N_{0}-1}=d(0)\right\} & =\left(1-p_{0,0}^{\left[N_{0}-2\right]}\right) \mathcal{P}\left\{D_{N_{0}-2}=d(0)\right\} .
\end{aligned}
$$

By induction, we get for all $\nu \in\left\{\hat{\nu} \in \mathbb{N}: 0 \leq \hat{\nu}<N_{0}\right\}$ that

$$
\mathcal{P}\left\{D_{\nu}=d(0)\right\}=\pi_{0}(d(0)) \prod_{m=0}^{\nu-1}\left(1-p_{0,0}^{[m]}\right),
$$

where we assume $\prod_{m=0}^{-1}(\cdot)=1$, and $N_{0}$ is the total number of the iteration wherein the algorithm goes from state $d(0)$ into $d(1)$. 
We now consider the case $k+1$, wherein for estimating $\mathcal{P}\left\{D_{k+1}=d(q)\right\}$ we have for each $k \in \mathbb{N}, q \in \mathbb{N}$ :

$$
\begin{aligned}
\left\{D_{k+1}=d(q)\right\}= & \left\{D_{k+1}=d(q), D_{k}=d(q)\right\} \\
& +\left\{D_{k+1}=d(q), D_{k}=d(q-1)\right\} .
\end{aligned}
$$

Then, from Eq. (3.7), we have

$$
\begin{aligned}
\mathcal{P}\left\{D_{k+1}=d(q)\right\}= & \mathcal{P}\left\{D_{k+1}=d(q), D_{k}=d(q)\right\} \\
& +\mathcal{P}\left\{D_{k+1}=d(q), D_{k}=d(q-1)\right\},
\end{aligned}
$$

which yields for each $k \in \mathbb{N}, q \in \mathbb{N}_{+}$:

$$
\begin{aligned}
\mathcal{P}\left\{D_{k+1}=d(q)\right\}= & \mathcal{P}\left\{D_{k+1}=d(q) \mid D_{k}=d(q)\right\} \mathcal{P}\left\{D_{k}=d(q)\right\} \\
& +\mathcal{P}\left\{D_{k+1}=d(q) \mid D_{k}=d(q-1)\right\} \mathcal{P}\left\{D_{k}=d(q-1)\right\} .
\end{aligned}
$$

Using Eqs. (3.1) and (3.2), we get for all $k \in \mathbb{N}, q \in \mathbb{N}_{+}$:

$$
\mathcal{P}\left\{D_{k+1}=d(q)\right\}=\left(1-p_{q, q}^{[k]}\right) \mathcal{P}\left\{D_{k}=d(q)\right\}+p_{q-1, q}^{[k]} \mathcal{P}\left\{D_{k}=d(q-1)\right\} .
$$

On the other hand, we know that for each $k \in \mathbb{N}, q \in \mathbb{N}_{+}$:

$$
\mathcal{P}\left\{D_{k}=d(q-1)\right\}+\mathcal{P}\left\{D_{k}=d(q)\right\}=1 .
$$

Replacing $\mathcal{P}\left\{D_{k}=d(q-1)\right\}$ of Eq. (3.9) into Eq. (3.8), we get

$$
\mathcal{P}\left\{D_{k+1}=d(q)\right\}=p_{q-1, q}^{[k]}+\left(1-p_{q, q}^{[k]}-p_{q-1, q}^{[k]}\right) \mathcal{P}\left\{D_{k}=d(q)\right\} .
$$

Substituting $k$ by $k+1$ in Eq. (3.10), we have

$$
\mathcal{P}\left\{D_{k+2}=d(q)\right\}=p_{q-1, q}^{[k+1]}+\left(1-p_{q, q}^{[k+1]}-p_{q-1, q}^{[k+1]}\right) \mathcal{P}\left\{D_{k+1}=d(q)\right\},
$$

what yields by replacing Eq. (3.10) into Eq. (3.11) for each $k \in \mathbb{N}, q \in \mathbb{N}_{+}$:

$$
\begin{aligned}
\mathcal{P}\left\{D_{k+2}=d(q)\right\}= & p_{q-1, q}^{[k+1]}+p_{q-1, q}^{[k]}\left(1-p_{q, q}^{[k+1]}-p_{q-1, q}^{[k+1]}\right) \\
& +\left(1-p_{q, q}^{[k+1]}-p_{q-1, q}^{[k+1]}\right)\left(1-p_{q, q}^{[k]}-p_{q-1, q}^{[k]}\right) \mathcal{P}\left\{D_{k}=d(q)\right\} .
\end{aligned}
$$

In general, we get for all $k \in \mathbb{N}, \nu \in \mathbb{N}_{+}, q \in \mathbb{N}_{+}$:

$$
\begin{aligned}
\mathcal{P}\left\{D_{k+\nu}=d(q)\right\}= & \sum_{n=0}^{\nu-1} p_{q-1, q}^{[k+n]} \prod_{m=n+1}^{\nu-1}\left(1-p_{q, q}^{[k+m]}-p_{q-1, q}^{[k+m]}\right) \\
& +\mathcal{P}\left\{D_{k}=d(q)\right\} \prod_{m=0}^{\nu-1}\left(1-p_{q, q}^{[k+m]}-p_{q-1, q}^{[k+m]}\right),
\end{aligned}
$$

where we have assumed that $\prod_{x}^{y}(\cdot)=1$, for all $x, y \in \mathbb{N}$ such that $x>y$.

The following lemma allows us to establish the fact that each state that belongs to $\{d(q)\}_{q=0}^{\infty}$ is non absorbing, therefore the process of identifying a local minimum will never stop, if the stopping rule is not met.

Lemma 3.6. Let $\{d(q)\}_{q=0}^{\infty}$ be the set of states visited by the MIRPSA. If the objective function is convex, then all $d(q)$ are non absorbing states. 
Proof. For proving the lemma, we must ensure that each $q$ th visited state can be left. In other words, $\mathcal{P}_{N^{[q]}+\nu}(d(q), d(q)) \neq 1$ and $\mathcal{P}_{N^{[q]}+\nu}(d(q), d(q+1)) \neq 0$, where $N^{[q]}$ given by $N^{[q]}=\sum_{i=0}^{q} N_{i}$ means the number of iterations carried out by the algorithm for arriving to each $q$ th state, and $\nu$ is the number of iterations performed by the algorithm within each $q$ th state.

From Eq. (3.6), we then have that for $M$ trial points

$$
\mathcal{P}_{N^{[q]}+\nu}(d(q), d(q))=\sum_{h=1}^{M}\left(\begin{array}{c}
M \\
h
\end{array}\right) \rho_{q, N[q]+\nu}^{h}\left(1-\rho_{q, N[q]}+\nu\right)^{M-h},
$$

where $\rho_{q, N^{[q]}+\nu}=\mathcal{P}\left\{\tilde{f}\left(z_{j}^{\left[q, N^{[q]}+\nu\right]}\right)<f\left(c^{\left[q, N^{[q]}+\nu\right]}\right)\right\}>0$ for each iteration due to convexity of the objective function.

This fact allows us to ensure that $\mathcal{P}_{N^{[q]}+\nu}(d(q), d(q))$ given by Eq. (3.14) is different from 1 for all $\nu \in \mathbb{N}$, and also, we can assert that

$$
\mathcal{P}_{N^{[q]}+\nu}(d(q), d(q+1))=\left(1-\rho_{q, N}[q]+\nu\right)^{M}>0, \quad \forall \nu \in \mathbb{N} .
$$

This last fact allows us to assure that the MIRPSA always goes from a $q$ th state to the $(q+1)$ th state with probability $\varphi(0<\varphi<1)$ until the stopping rule of the MIRPSA has been held. In our algorithm, the stopping rule is given by $d(k)=\delta^{[k]}+\Delta^{[k]}<\varepsilon$, for some $\varepsilon>0$.

Theorem 3.7. Assume that the MIRPSA is applied to a convex objective function, and let $\{d(q)\}_{q=0}^{\infty}$ be the set of visited states by the MIRPSA. If a moving operation is carried out at a qth state, then the MIRPSA will improve the objective function.

Proof. According to the algorithm, a moving operation at a $k$ th iteration causes the updating of the pattern search center by replacing $c_{k+1}$ by the best trial points in the current mixed integer randomized pattern search $\mathcal{H}_{k} \in \mathbb{R}^{n} \times \mathbb{Z}^{m}$, what allows us to assert that $f\left(c_{k+1}\right)<f\left(c_{k}\right)$.

Theorem 3.8. If the MIRPSA is applied to a convex objective function, then the MIRPSA converges to a local minimum at least.

Proof. Since the MIRPSA visits all $q$ th non absorbing state $d(q)$ in $\{d(q)\}_{q=0}^{\infty}$ due to Lemma 3.6, and according to Proposition 3.4 the probability of a moving operation taking place is non zero, then an improvement of the objective function occurs due to Theorem 3.7 The MIRPSA therefore converges to a local minimum at least, when infinite states are visited.

At this stage we have proved that the MIRPSA allows us to identify a local minimum at least. However, we did not provide a theoretical study of the convergence rate of the MIRPSA, which will here be proposed by the next theorem and associated corollary. It is worthwhile to remark that the convex hull volume of each mixed integer randomized pattern search $\mathcal{H}_{k} \in \mathbb{R}^{n} \times \mathbb{Z}^{m}$, at each $q$ th state, allows us to prove the convergence rate of the MIRPSA. 
Theorem 3.9. Assume that there exist $\Delta^{[0]}>1$ and $\beta \in \mathbb{R}$ such that $0<\beta<1$, and also suppose that the $n+m$ dimensional volume $v(q)$ of the convex hull $C\left(\mathcal{H}_{q}\right)$ at each qth state is given, according to Remark 3.2, by Eq. (3.4). Then, for any state counter $q \in \mathbb{N}$,

$$
\frac{v(q+1)}{v(q)}= \begin{cases}\alpha^{n} \beta^{m}, & 0 \leq q<\tilde{q}-1 \\ \frac{\alpha^{n}}{\beta^{m} \tilde{q} \Delta^{[0]^{m}},} & q=\tilde{q}-1 \\ \alpha^{n}, & q \geq \tilde{q}\end{cases}
$$

where $\tilde{q}=\left\lfloor\frac{-\ln \left(\Delta^{[0]}\right)}{\ln (\beta)}\right\rfloor$.

Proof. Since

$$
\max \left(1, \Delta^{[q]}\right)= \begin{cases}\Delta^{[q]}, & \Delta^{[q]}>1 \\ 1, & \Delta^{[q]} \leq 1\end{cases}
$$

Eq. (3.16) can be rewritten as

$$
\max \left(1, \Delta^{[q]}\right)= \begin{cases}\beta^{q} \Delta^{[0]}, & q<\frac{-\ln \left(\Delta^{[0]}\right)}{\ln (\beta)} \\ 1, & q \geq \frac{-\ln \left(\Delta^{[0]}\right)}{\ln (\beta)}\end{cases}
$$

because of $0<\beta<1$.

On the other hand, according to Remark 3.2 and taking into account Eq. (3.17), we have that the volume of each convex hull $C\left(\mathcal{H}_{q}\right)$ in the Euclidean space $\mathbb{R}^{n} \times \mathbb{N}^{m}$ at each $q$ th state is given by

$$
v(q)= \begin{cases}2^{n+m}\left(\alpha^{q} \delta^{[0]}\right)^{n}\left(\beta^{q} \Delta^{[0]}\right)^{m}, & 0 \leq q<\frac{-\ln \left(\Delta^{[0]}\right)}{\ln (\beta)} \\ 2^{n+m}\left(\alpha^{q} \delta^{[0]}\right)^{n}, & q \geq \frac{-\ln \left(\Delta^{[0]}\right)}{\ln (\beta)}\end{cases}
$$

where we have assumed that $\Delta^{[0]}>1$ and $0<\beta<1$.

From Eq. (3.18), we can assure that

$$
\frac{v(q+1)}{v(q)}= \begin{cases}\alpha^{n} \beta^{m}, & 0 \leq q<\tilde{q}-1 \\ \frac{\alpha^{n}}{\beta^{m} \tilde{q} \Delta^{[0]^{m}},} & q=\tilde{q}-1 \\ \alpha^{n}, & q \geq \tilde{q}\end{cases}
$$

where $\tilde{q}=\left\lfloor\frac{-\ln \left(\Delta^{[0]}\right)}{\ln (\beta)}\right\rfloor$.

Of course, it is not difficult to verify that if there exists a $\tilde{q}$ such that $\frac{-\ln \left(\Delta^{[0]}\right)}{\ln (\beta)} \in$ $\mathbb{N}$, then, for this particular case, Eq. (3.19) can be rewritten as

$$
\frac{v(q+1)}{v(q)}= \begin{cases}\alpha^{n} \beta^{m}, & 0 \leq q<\tilde{q}-1 \\ \alpha^{n}, & q \geq \tilde{q}-1\end{cases}
$$

due to the fact that $\beta^{\tilde{q}} \Delta^{[0]}=1$ 
Corollary 3.10. Assume that there exist $\Delta^{[0]}>1$ and $\beta \in \mathbb{R}$ such that $0<\beta<1$. If $0<\alpha<1$, then the volume $v(q)$ of the convex hull $C\left(\mathcal{H}_{q}\right)$ shrinks at a rate of $\alpha^{n} \beta^{m}$, for all $0 \leq q<\tilde{q}-1$, or $\alpha^{n}$, for all $q \geq \tilde{q}-1$, and therefore $\lim _{q \rightarrow \infty} v(q)=0$.

Proof. From Eq. (3.19), we have that

$$
\frac{v(q+1)}{v(q)} \propto \begin{cases}\alpha^{n} \beta^{m}, & 0 \leq q<\tilde{q}-1 \\ \alpha^{n}, & q \geq \tilde{q}-1,\end{cases}
$$

which obviously allows us to assure that

$$
v(q) \propto \begin{cases}\left(\alpha^{n} \beta^{m}\right)^{q}, & 0 \leq q<\tilde{q}-1 ; \\ \alpha^{n q}, & q \geq \tilde{q}-1,\end{cases}
$$

where $\tilde{q}=\left\lfloor\frac{-\ln \left(\Delta^{[0]}\right)}{\ln (\beta)}\right\rfloor$, and the volume $v(q)$ of the convex hull $C\left(\mathcal{H}_{q}\right)$ therefore becomes smaller in volume at a rate of $\alpha^{n} \beta^{m}$, for all $0 \leq q<\tilde{q}-1$, or $\alpha^{n}$, for all $q \geq \tilde{q}-1$. As a result of this, we can assert that the rate of shrinking is degenerated by increasing $n$ and $m$.

Furthermore, from Eq. (3.20), it can be concluded that

$$
\lim _{q \rightarrow \infty} v(q)=0,
$$

because we have assumed that $\Delta^{[0]}>1,0<\beta<1$, and $0<\alpha<1$.

\section{A LOW DIMENSION CASE}

In what follows, we analyze a low dimension case, $\mathbb{R} \times \mathbb{Z}$. This could be considered the simplest case; it is, however, a nontrivial case.

4.1. Estimating $\rho_{q, k+\nu}=\mathcal{P}\left\{\tilde{f}\left(z_{j}^{[q, k]}\right)<f\left(c^{[q, k]}\right)\right\}$. In order to simplify our notation and without loss of generality, we shall denote $z^{[q, k]}$ and $c^{[q, k]}$ without the superscript $[q, k]$.

Note that the measure of the randomized pattern search $\mathcal{H}_{k}$ is equal to $\mathcal{H}_{k+\nu}$, because the MIRPSA is at the $q$ th state for $\nu$ iterations.

Let $W$ be a random variable given by a convex function $\tilde{f}(v): \mathbb{R} \times \mathbb{Z} \rightarrow \mathbb{R}$, which is defined by

$$
\tilde{f}(v)=b_{0}+\tilde{v}^{t} b+\tilde{v}^{t} A \tilde{v}, \quad \forall v \in \mathbb{R} \times \mathbb{Z}
$$

where $\tilde{v}=(\tilde{x}, \tilde{y})^{t} \in \mathbb{R} \times \mathbb{Z}$ is a random vector, because $\tilde{x}$ is distributed by a continuous uniform density function $\mathrm{u}\left(-\delta^{[k]}, \delta^{[k]}\right)$, for each $k$ th iteration $\delta^{[k]} \in \mathbb{R}_{+}$; $\tilde{y}$ is also distributed by a discrete uniform density function $\mathrm{u}\left(-\Delta^{[k]}, \Delta^{[k]}\right)$, for each $k$ th iteration $\Delta^{[k]} \in \mathbb{N}_{+} ; b_{0} \in \mathbb{R}$; the vector $b=\left(b^{(1)}, b^{(2)}\right)^{t} \in \mathbb{R}^{2 \times 1} ;$ and the matrix $A=\left(\begin{array}{ll}a^{(11)} & a^{(12)} \\ a^{(21)} & a^{(22)}\end{array}\right)$, which is a positive definite matrix in $\mathbb{R}^{2 \times 2}$, because we have assumed that our objective function is convex.

Note that the stationary point of the surface is given by $\hat{v}=\left(\hat{v}^{(1)}, \hat{v}^{(2)}\right)^{t}=$ $-\frac{1}{2} A^{-1} b$, which does not necessarily belong to Euclidean space $\mathbb{R} \times \mathbb{Z}$ [10, Ch. 6].

As a consequence of these facts, we then have a random function $\tilde{w}$ of two random variables $(\tilde{x}, \tilde{y})^{t} \in \mathbb{R} \times \mathbb{Z}$ given by $\tilde{w}=b_{0}+v^{t} b+v^{t} A v$. 
According to the MIRPSA, at each $k$ th iteration, the objective function value at random pattern search center $c=\left(c^{(1)}, c^{(2)}\right)^{t}$ is known as a result of either moving operation or shrinking operation at the $(k-1)$ th iteration, and its deterministic value is $f(c)$. Note that at each $k$ th iteration, the MIRPSA obtains a $k$ th pattern search center, which will be denoted by $c$ without the superscript $[k]$ in order to simplify the notation. In addition, we have a set of $M$ trial points $z_{j}$ and each $j$ th random trial point with a value given by $\tilde{f}\left(z_{j}\right)$.

At each $k$ th iteration, we can have two main situations: the first one when the stationary point $\hat{v}$ of the objective function is outside the $k$ th random pattern search, and the second situation is when it is inside the $k$ th pattern search $\mathcal{H}_{k}$.

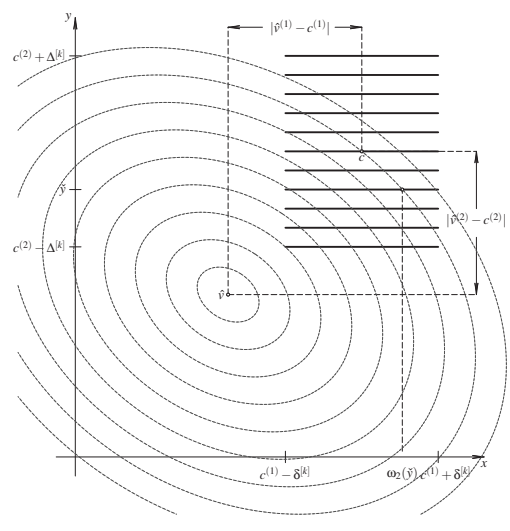

(a) The center of the pattern is far away from the stationary point $\hat{v}$.

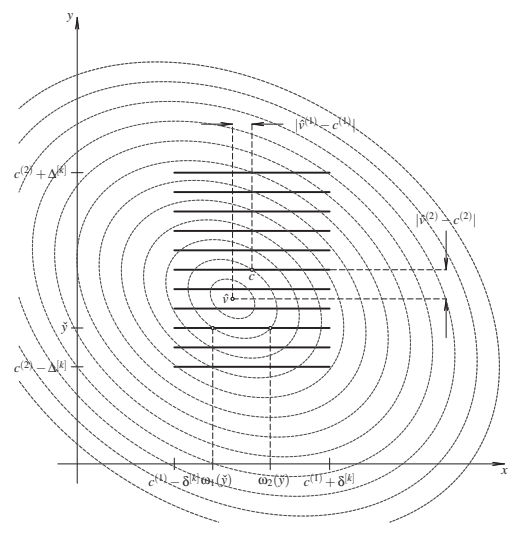

(b) The center of the pattern is near the stationary point $\hat{v}$.

FiguRE 3. Example pattern search in $\mathbb{R} \times \mathbb{Z}$ for the cases (a) when the center of the pattern is far away from the stationary point, and (b) when the center of the pattern is near the stationary point.

Consider Fig. 3(a) which displays a $k$ th pattern search of size $4 \delta^{[k]} \Delta^{[k]}$ in $\mathbb{R} \times \mathbb{Z}$, with center at $c=\left(c^{(1)}, c^{(2)}\right)^{t}$. In addition, it shows the stationary point of the objective function surface $\hat{v}=\left(\hat{v}^{(1)}, \hat{v}^{(2)}\right)^{t}$ on the plane $x y$, which is not necessarily a minimum of our problem, and the center of the pattern is far away from the vertex $\hat{v}$.

Meanwhile, a case when the center of the pattern is near the stationary point of the objective function surface $\hat{v}$ is depicted in Fig. 3(b). The figure also shows on the $x$ axis the points $\omega_{1}(\check{y})$ and $\omega_{2}(\check{y})$, whose difference lets us estimate the probability $\mathcal{P}\left\{\tilde{f}\left(z_{j}\right)<f(c)\right\}$ for the particular case $(x, \breve{y})^{t}$.

It is worthwhile pointing out that both figures depict a set of level curves of the generic mixed integer convex quadratic objective function, because its integer variable $y$ was converted to a real variable for graphically showing the objective function. 
Under the above enunciated considerations, we can assert

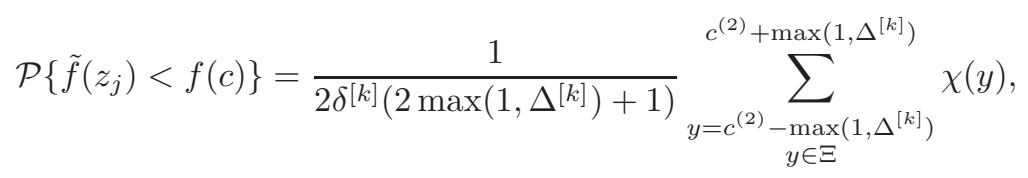

where

$$
\begin{gathered}
\chi(y)=\min \left(c^{(1)}+\delta^{[k]}, \omega_{2}(y)\right)-\max \left(c^{(1)}-\delta^{[k]}, \omega_{1}(y)\right) \\
\omega_{1}(y)=\frac{-\left(b^{(1)}+\left(a^{(12)}+a^{(21)}\right) y\right)}{2 a^{(11)}}-\frac{\sqrt{\psi\left(b_{0}, b, A\right)}}{2 a^{(11)}} \\
\omega_{2}(y)=\frac{-\left(b^{(1)}+\left(a^{(12)}+a^{(21)}\right) y\right)}{2 a^{(11)}}+\frac{\sqrt{\psi\left(b_{0}, b, A\right)}}{2 a^{(11)}} \\
\left.\psi\left(b_{0}, b, A\right)=b^{(1)}+\left(a^{(12)}+a^{(21)}\right) y\right)^{2}-4 a^{(11)}\left(b_{0}+b^{(2)} y+a^{(22)} y^{2}-f\left(c^{[k]}\right),\right. \\
f\left(c^{[k]}\right)=b_{0}+c^{t} b+c^{t} A c \\
\Xi=\left\{n \in \mathbb{Z}: \psi\left(b_{0}, b, A\right) \geq 0\right\} .
\end{gathered}
$$

From Eq. (4.2) we easily get

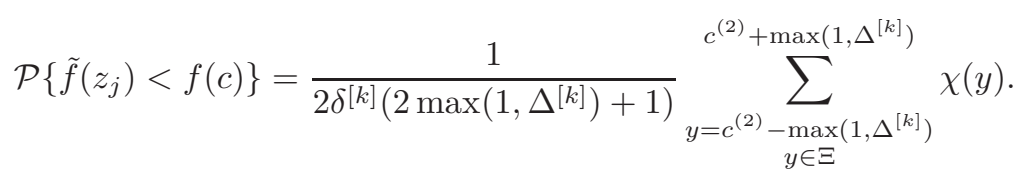
have

Therefore, since $\mathcal{P}\left\{\tilde{f}\left(z_{j}\right)<f(c)\right\}+\mathcal{P}\left\{\tilde{f}\left(z_{j}\right) \geq f(c)\right\}=1$ for all $k \in \mathbb{N}$, we also

$$
\mathcal{P}\left\{\tilde{f}\left(z_{j}\right) \geq f(c)\right\}=1-\frac{1}{2 \delta^{[k]}\left(2 \max \left(1, \Delta^{[k]}\right)+1\right)} \sum_{y=c^{(2)}}^{c_{-\max \left(1, \Delta^{[k]}\right)}^{(2)}} \chi(y) .
$$

4.2. A numerical example. Let $\tilde{w}$ be a random variable given by

$$
\tilde{w}=\tilde{f}(v)=\tilde{x}^{2}+\tilde{y}^{2}, \quad \forall v \in \mathbb{R} \times \mathbb{Z},
$$

where $\tilde{v}=(\tilde{x}, \tilde{y})^{t} \in \mathbb{R} \times \mathbb{Z}$ is a random vector, because $\tilde{x}$ is distributed by a continuous uniform density function $\mathrm{u}\left(-\delta^{[k]}, \delta^{[k]}\right)$, for each $k$ th iteration $\delta^{[k]} \in \mathbb{R}_{+}$; and $\tilde{y}$ is also distributed by a discrete uniform density function $\mathrm{u}\left(-\Delta^{[k]}, \Delta^{[k]}\right)$, for each $k$ th iteration $\Delta^{[k]} \in \mathbb{N}_{+}$. As a result of this fact, we have a random function $W$ of two random variables in $\mathbb{R} \times \mathbb{Z}$.

We also say that in our example the parameters $b_{0}=b^{(1)}=b^{(2)}=0$ and $a^{(12)}=a^{(21)}=0$. Meanwhile, the parameters of the quadratic function given by Eq. (4.1) are $a^{(11)}=a^{(22)}=1$. Therefore, using Eqs. (4.4a) and 4.4b), we get

$$
\omega_{1}(y)=-\sqrt{f\left(c^{[k]}\right)-y^{2}} ; \quad \omega_{2}(y)=\sqrt{f\left(c^{[k]}\right)-y^{2}} .
$$


This allows us to estimate the probability $\mathcal{P}\left\{\tilde{f}\left(z_{j}\right)<f(c)\right\}$ using Eq. (4.2), that is, the probability of carrying out a moving operation at any $k$ th iteration, which is therefore given by

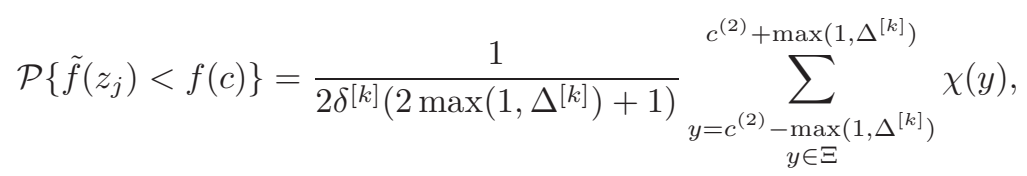

where for this particular example

$$
\Xi=\left\{n \in \mathbb{Z}:|n| \leq+\sqrt{f\left(c^{[k]}\right)}\right\},
$$

and $\chi(y)$ is calculated using Eqs. (4.3) and (4.5).

According to the MIRPSA, at the $k$ th iteration, the objective function value at random pattern search center $c$ is known by both moving operation or shrinking operation carried out at the $(k-1)$ th iteration, and its deterministic value is $\tilde{f}(c)=$ $f_{0}^{[k]}$. In addition, we have a set of $M$ trial points $z_{j}$ and each $j$ th random trial point with a value given by $\tilde{f}\left(z_{j}\right)$.

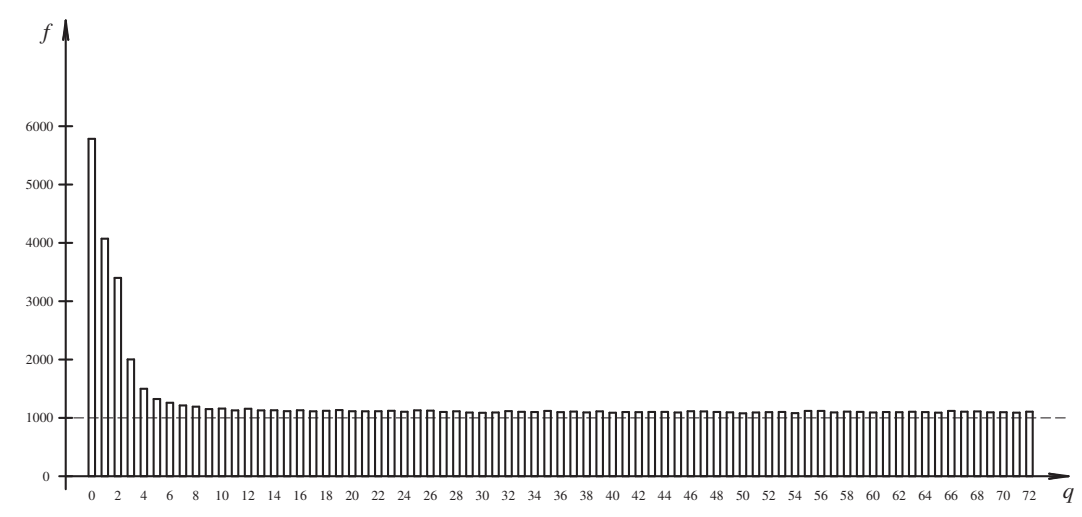

Figure 4. Frequency histogram for each $q$ th state with 1000 replications

Fig. 4 shows a frequency histogram for each $q$ th state over an experiment of 1000 independent replications, and the parameter values of the MIRPSA, for $n=m=1$, were: $\alpha=0.9, \delta=2, \beta=0.6, \Delta=5, \varepsilon=10^{-3}$, and two samples per dimension, with an initial pattern search center $v_{0}=(10,10)^{t}$. According to the figure, a total of 73 states were visited 1000 times at least by the MIRPSA. On the other hand, it can be seen from the figure that the imaginary envelope curve to the frequencies quickly flattens out.

\section{ON THE PERFORMANCE OF THE MIRPSA}

Consider the test problem shown in A.1 (Appendix A), which is a mixed integer symmetrical quadratic function. 
Fig. [5 shows the average value of the mixed integer quadratic function given by Eq. A.1 in Section A.1. for the cases: $\mathbb{R}^{n} \times \mathbb{Z}^{m}$ where $n=m$ and $m \in$ $\{5,10,15,20\}$; at each $k$ th iteration, for 30 replications; when the parameter values of the MIRPSA were fixed at: $\alpha=0.9 ; \delta=2 ; \beta=0.6 ; \Delta=5 ; \varepsilon=10^{-3}$, an initial point $v_{0}^{(i)}=10$ for all $i=1, \ldots, n+m$, and a number random trial points $M=2(n+m)$.

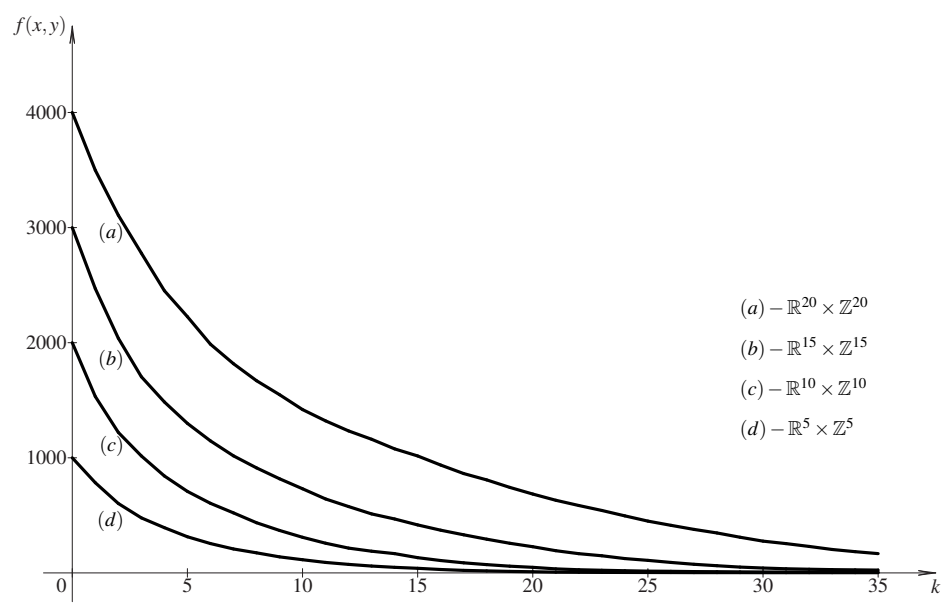

FiguRE 5. Average of Quadratic function value by iteration

As can be seen from the graph, the rate of convergence for this particular case depends on the dimension of the function, and the average of objective function, for each case (a), (b), (c), and (d), shows a downward trend.

5.1. More numerical examples. A set of additional numerical examples are shown here. Each test problem was carried out with: 30 replications; number of function evaluations equal to $100(n+m)^{2}$; and the parameters of the MIRPSA were fixed at $\alpha=0.9 ; \delta=5 ; \beta=0.99$ and $\Delta=5$, for a sample of 2 trail points per dimension.

All the initial points for the test problems of Appendix A were at 10 for each component, except for the test problem MRF, which was an initial point with each component fixed at 4 .

The following tables report: mean $(\hat{\mu})$, standard deviation $(\hat{\sigma})$ and range of both $m(p, s)$ and distance to the true point (DTP) for a set of test problems, which are formulated in Appendix A

As can be seen from Table 1, the MIRPSA had an adequate $m(p, s)$ for the case of the test problem QF, because we get a mean value between $10^{-7}$ and $10^{-3}$. Moreover, we obtained a small DTP mean, which yield values between $10^{-3}$ and 4 for all dimensional cases, if taking into account the NE for each case. 
TABLE 1. Results of numerical example QF

\begin{tabular}{|c|c|c|c|c|c|c|c|c|}
\hline \multirow[b]{2}{*}{$n$} & \multirow[b]{2}{*}{$m$} & \multirow[b]{2}{*}{$\mathrm{NE}$} & \multicolumn{3}{|c|}{$m(p, s)$} & \multicolumn{3}{|c|}{ DTP } \\
\hline & & & $\hat{\mu}$ & $\hat{\sigma}$ & Range & $\hat{\mu}$ & $\hat{\sigma}$ & Range \\
\hline 2 & 2 & 1600 & $4.887 \mathrm{E}-7$ & $2.354 \mathrm{E}-6$ & $1.285 \mathrm{E}-5$ & $4.063 \mathrm{E}-3$ & $1.361 \mathrm{E}-2$ & $7.161 \mathrm{E}-2$ \\
\hline 2 & 5 & 4900 & $2.731 \mathrm{E}-4$ & $2.842 \mathrm{E}-4$ & $1.208 \mathrm{E}-3$ & $3.793 \mathrm{E}-1$ & $2.212 \mathrm{E}-1$ & $9.053 \mathrm{E}-1$ \\
\hline 5 & 2 & 4900 & $1.839 \mathrm{E}-9$ & 8.870E-9 & $4.865 \mathrm{E}-8$ & $1.081 \mathrm{E}-5$ & $3.480 \mathrm{E}-5$ & $1.845 \mathrm{E}-4$ \\
\hline 5 & 5 & 10000 & $8.955 \mathrm{E}-7$ & $6.793 \mathrm{E}-7$ & $3.108 \mathrm{E}-6$ & $8.854 \mathrm{E}-4$ & $3.399 \mathrm{E}-4$ & $1.609 \mathrm{E}-3$ \\
\hline 5 & 10 & 22500 & $1.431 \mathrm{E}-6$ & $6.061 \mathrm{E}-7$ & $2.063 \mathrm{E}-6$ & $1.437 \mathrm{E}-3$ & $2.909 \mathrm{E}-4$ & $9.948 \mathrm{E}-4$ \\
\hline 10 & 5 & 22500 & $1.125 \mathrm{E}-6$ & $5.373 \mathrm{E}-7$ & $2.153 \mathrm{E}-6$ & $1.263 \mathrm{E}-3$ & $3.100 \mathrm{E}-4$ & $1.296 \mathrm{E}-3$ \\
\hline 10 & 10 & 40000 & $2.100 \mathrm{E}-6$ & $8.211 \mathrm{E}-7$ & $3.456 \mathrm{E}-6$ & $2.015 \mathrm{E}-3$ & $3.802 \mathrm{E}-4$ & $1.612 \mathrm{E}-3$ \\
\hline 10 & 15 & 62500 & $2.725 \mathrm{E}-3$ & $8.043 \mathrm{E}-4$ & $3.140 \mathrm{E}-3$ & 2.580331 & 0.400351 & 1.587830 \\
\hline 15 & 10 & 62500 & $2.203 \mathrm{E}-3$ & $8.986 \mathrm{E}-4$ & $4.082 \mathrm{E}-3$ & 2.304014 & 0.452954 & 2.015951 \\
\hline 15 & 15 & 90000 & $3.037 \mathrm{E}-3$ & $9.196 \mathrm{E}-4$ & $3.330 \mathrm{E}-3$ & 2.986169 & 0.446884 & 1.624471 \\
\hline 20 & 20 & 160000 & $4.028 \mathrm{E}-3$ & $8.636 \mathrm{E}-4$ & $3.719 \mathrm{E}-3$ & 3.991904 & 0.428823 & 1.841649 \\
\hline
\end{tabular}

Table2 depicts the obtained results of the MIRPSA for the test problem McMF. As is shown in the table, the MIRPSA adequately identified the optimum solution for each dimensional case.

TABLE 2. Results of numerical example McKF

\begin{tabular}{|c|c|c|c|c|c|c|c|c|}
\hline \multirow[b]{2}{*}{$n$} & \multirow[b]{2}{*}{$m$} & \multirow[b]{2}{*}{$\mathrm{NE}$} & \multicolumn{3}{|c|}{$m(p, s)$} & \multicolumn{3}{|c|}{ DTP } \\
\hline & & & $\hat{\mu}$ & $\hat{\sigma}$ & Range & $\hat{\mu}$ & $\hat{\sigma}$ & Range \\
\hline 2 & 2 & 1600 & $3.198 \mathrm{E}-5$ & $8.943 \mathrm{E}-5$ & $3.818 \mathrm{E}-4$ & $3.969 \mathrm{E}-2$ & $7.872 \mathrm{E}-2$ & $3.003 \mathrm{E}-1$ \\
\hline 2 & 5 & 4900 & $4.403 \mathrm{E}-4$ & $5.854 \mathrm{E}-4$ & $2.111 \mathrm{E}-3$ & $2.528 \mathrm{E}-1$ & $2.022 \mathrm{E}-1$ & $7.762 \mathrm{E}-1$ \\
\hline 5 & 2 & 4900 & $2.258 \mathrm{E}-5$ & $6.552 \mathrm{E}-5$ & $3.344 \mathrm{E}-4$ & $6.610 \mathrm{E}-2$ & $8.952 \mathrm{E}-2$ & $3.344 \mathrm{E}-4$ \\
\hline 5 & 5 & 10000 & $5.692 \mathrm{E}-4$ & $3.194 \mathrm{E}-4$ & $1.037 \mathrm{E}-3$ & $5.343 \mathrm{E}-1$ & $1.721 \mathrm{E}-1$ & $6.168 \mathrm{E}-1$ \\
\hline 5 & 10 & 22500 & $1,273 \mathrm{E}-3$ & $5.899 \mathrm{E}-4$ & $2.694 \mathrm{E}-3$ & 1.157214 & $2.764 \mathrm{E}-1$ & 1.151966 \\
\hline 10 & 5 & 22500 & $8,309 \mathrm{E}-4$ & $4,599 \mathrm{E}-4$ & $2,335 \mathrm{E}-3$ & $9,023 \mathrm{E}-1$ & $2,522 \mathrm{E}-1$ & 1,299292 \\
\hline 10 & 10 & 40000 & $1.285 \mathrm{E}-3$ & $4.089 \mathrm{E}-4$ & $1.681 \mathrm{E}-3$ & 1.371443 & $2.422 \mathrm{E}-1$ & 1.036460 \\
\hline 10 & 15 & 62500 & $1.886 \mathrm{E}-3$ & $5.474 \mathrm{E}-4$ & $1.853 \mathrm{E}-3$ & 1.973490 & 0.230806 & 1.006717 \\
\hline 15 & 10 & 62500 & $1.463 \mathrm{E}-3$ & $4.105 \mathrm{E}-4$ & $1.726 \mathrm{E}-3$ & 1.636571 & 0,248927 & 1.121923 \\
\hline 15 & 15 & 90000 & $2.226 \mathrm{E}-3$ & $6.701 \mathrm{E}-4$ & $2.559 \mathrm{E}-3$ & 2.303539 & 0.265055 & 1.129471 \\
\hline 20 & 20 & 160000 & $2.606 \mathrm{E}-3$ & $6.481 \mathrm{E}-4$ & $2.857 \mathrm{E}-3$ & 3.003909 & 0.298459 & 1.309361 \\
\hline
\end{tabular}

A report of the mixed integer Extended Rosenbrock Function test problem (see Appendix A) is shown in Table 3, which allows us to assert that the MIRPSA achieved a good performance for all dimensional cases.

Finally, Table 4 displays the performance results of the test problem ADF, defined in Appendix A As we can see from Table 4, the MIRPSA identified the optimum solution with 900 function evaluations, and a mean of $m(p, s)=1.570 \mathrm{E}-6$.

\section{Conclusions And Future Research}

In this article, we have examined some convergence properties of the MIRPSA based on the approach of Markov chains. We have proved that the MIRPSA visits 
TABLE 3. Results of numerical example ERF

\begin{tabular}{|c|c|c|c|c|c|c|c|c|}
\hline \multirow[b]{2}{*}{$n$} & \multirow[b]{2}{*}{$m$} & \multirow[b]{2}{*}{$\mathrm{NE}$} & \multicolumn{3}{|c|}{$m(p, s)$} & \multicolumn{3}{|c|}{ DTP } \\
\hline & & & $\hat{\mu}$ & $\hat{\sigma}$ & Range & $\hat{\mu}$ & $\hat{\sigma}$ & Range \\
\hline 2 & 2 & 1600 & $2.181 \mathrm{E}-4$ & $2.384 \mathrm{E}-4$ & $7.322 \mathrm{E}-4$ & 4.88664 & 3.98084 & 15.2164 \\
\hline 4 & 4 & 6400 & $6.376 \mathrm{E}-4$ & $6.570 \mathrm{E}-4$ & $2.597 \mathrm{E}-3$ & 9.67566 & 4.59177 & 17.6454 \\
\hline 6 & 6 & 14400 & $1.755 \mathrm{E}-3$ & $2.081 \mathrm{E}-3$ & $9.332 \mathrm{E}-3$ & 13.4318 & 4.62141 & 18.2798 \\
\hline 8 & 8 & 25600 & $3.273 \mathrm{E}-3$ & $3.202 \mathrm{E}-3$ & $1.316 \mathrm{E}-2$ & 15.0772 & 4.19551 & 17.5384 \\
\hline 10 & 10 & 40000 & $5.375 \mathrm{E}-3$ & $5.743 \mathrm{E}-3$ & $2.734 \mathrm{E}-2$ & 16.0987 & 4.48759 & 17.4839 \\
\hline 14 & 14 & 78400 & $1.126 \mathrm{E}-2$ & $9.302 \mathrm{E}-3$ & $4.660 \mathrm{E}-2$ & 22.1805 & 4.43726 & 19.9428 \\
\hline 18 & 18 & 129600 & $1.321 \mathrm{E}-2$ & $6.306 \mathrm{E}-3$ & $2.895 \mathrm{E}-2$ & 22.7375 & 3.77082 & 15.9504 \\
\hline 20 & 20 & 160000 & $1.683 \mathrm{E}-2$ & $1.006 \mathrm{E}-2$ & $4.170 \mathrm{E}-2$ & 25.0446 & 4.99112 & 24.3979 \\
\hline
\end{tabular}

TABLE 4. Results of numerical example ADF

\begin{tabular}{|c|c|c|c|c|c|c|c|c|}
\hline \multirow[b]{2}{*}{$n$} & \multirow[b]{2}{*}{$m$} & \multirow[b]{2}{*}{$\mathrm{NE}$} & \multicolumn{3}{|c|}{$m(p, s)$} & \multicolumn{3}{|c|}{ DTP } \\
\hline & & & $\hat{\mu}$ & $\hat{\sigma}$ & Range & $\hat{\mu}$ & $\hat{\sigma}$ & Range \\
\hline 2 & 1 & 900 & $1.570 \mathrm{E}-6$ & $3.417 \mathrm{E}-6$ & $1.127 \mathrm{E}-5$ & 0.470910 & 1.205474 & 3.992517 \\
\hline
\end{tabular}

each $q$ th state, and we have also proved that the MIRPSA has non absorbing states, which guarantees that the process of finding a local minimum stops when the stopping rule is satisfied, namely, only if $\alpha^{k} \delta^{[0]}+\beta^{k} \Delta^{[0]} \leq \varepsilon$ is satisfied, the MIRPSA will stop at $k$ th iteration, and the MIRPSA therefore at least identifies a local minimum.

Future research will focus on analyzing and tuning the MIRPSA using the approach of design of experiments. We also suggest to include some backtracking rules to the MIRPSA in order to study the performance of a modified MIRPSA for identifying global minimum of unconstrained nonlinear mixed integer problems.

\section{Appendix A. Test problems}

Here we present a set of mixed integer test problems, wherein we show the optimum solution of each test problem.

\section{A.1. Mixed integer quadratic function: QF.}

$$
f(x, y)=\sum_{i=1}^{n} a^{(i)} x^{(i)^{2}}+\sum_{j=1}^{m} b^{(j)} y^{(j)^{2}}, \quad \forall x \in \mathbb{R}^{n}, y \in \mathbb{Z}^{m},
$$

where $a^{(i)} \in \mathbb{R}$ and $b^{(i)} \in \mathbb{R}$ are all positive coefficients for all $i=1, \ldots, n$, and $j=1, \ldots, m$. In this paper $a^{(i)}$ and $b^{(i)}$ were all fixed at 1 .

Solution. The global minimum is located at $\hat{x}^{t}=(\underbrace{0,0, \ldots, 0}_{n})$ and $\hat{y}^{t}=(\underbrace{0,0, \ldots, 0}_{m})$ with value function $f(\hat{v})=0$. 
A.2. Mixed integer McKinnon function: McKF. We show here a modified variant of the original McKinnon function [9].

$$
f(x, y)=\sum_{i=1}^{n} \lambda^{(i)} x^{(i)^{2}}+\sum_{i=1}^{m}\left|y^{(i)}\right|+y^{(i)^{2}}, \quad \forall x \in \mathbb{R}^{n}, y \in \mathbb{Z}^{m}
$$

where

$$
\lambda^{(i)}= \begin{cases}6, & \forall x^{(i)} \geq 0 \\ 360, & \forall x^{(i)}<0\end{cases}
$$

Solution. The global minimum is located at $\hat{x}^{t}=(\underbrace{0,0, \ldots, 0}_{n})$ and $\hat{y}^{t}=(\underbrace{0,0, \ldots, 0}_{m})$ with value function $f(\hat{v})=0$.

\section{A.3. Mixed integer extended Rosenbrock function: ERF.}

$$
f(x, y)=\sum_{i=1}^{d}\left(\left(x^{(i)}-y^{(i)^{2}}\right)^{2}+\left(1-y^{(i)}\right)^{2}\right), \quad \forall x \in \mathbb{R}^{d}, y \in \mathbb{Z}^{d},
$$

where the dimension of the problem, denoted by $d$, is an even positive integer number.

Solution. The global minimum is located at $\hat{x}^{t}=(\underbrace{1,1, \ldots, 1}_{d})$ and $\hat{y}^{t}=(\underbrace{1,1, \ldots, 1}_{d})$ with a function value of $f(\hat{v})=0$.

A.4. Audet \& Dennis function: ADF. This problem, proposed by Audet and Dennis [1], is given by

$$
\underset{(x, y) \in \mathbb{R}^{2} \times \mathbb{Z}}{\operatorname{minimize}} f(x, y)
$$

subject to

$$
-2 \leq x^{(1)} \leq 2 ; \quad-2 \leq x^{(2)} \leq 2 ; \quad y^{(1)} \in\{0,1\},
$$

where

$$
f(x, y)=g\left(x^{(1)}, x^{(2)}\right)\left(1-y^{(1)}\right)+h\left(x^{(1)}, x^{(2)}\right) y^{(1)}, \quad \forall x \in \mathbb{R}^{2}, y \in \mathbb{Z} ;
$$

and the functions $g\left(x^{(1)}, x^{(2)}\right)$ and $h\left(x^{(1)}, x^{(2)}\right)$ for all $x \in \mathbb{R}^{2}$ are given by

$$
\begin{aligned}
& g\left(x^{(1)}, x^{(2)}\right)=x^{(1)^{2}}+x^{(2)^{2}} ; \\
& h\left(x^{(1)}, x^{(2)}\right)=x^{(1)^{2}} x^{(2)}+x^{(1)}\left(1-x^{(2)}\right) .
\end{aligned}
$$

Using the penalty function approach, this problem has been rewritten, for all $x \in \mathbb{R}^{2}$ and $y \in \mathbb{Z}$, as

$$
f(x, y)=g\left(x^{(1)}, x^{(2)}\right)\left(1-y^{(1)}\right)+h\left(x^{(1)}, x^{(2)}\right) y^{(1)}+\sum_{i=1}^{2} p_{i}\left(x^{(i)}\right)+p_{3}\left(y^{(1)}\right)
$$


where, for $k=10^{3}$,

$$
\begin{aligned}
& p_{i}\left(x^{(i)}\right)=k \max \left(-2-x^{(i)}, 0\right)+k \max \left(x^{(i)}-2,0\right), \quad x^{(i)} \in \mathbb{R} ; \\
& p_{3}\left(y^{(1)}\right)=k \max \left(-y^{(1)}, 0\right)+k \max \left(y^{(1)}-1,0\right), \quad y^{(1)} \in \mathbb{Z} .
\end{aligned}
$$

Solution. The global minimum is $\hat{x}^{t}=(-2,-2)$ and $\hat{y}=(1)$, with minimum value function $f(\hat{v})=-14$.

\section{ACKNOWLEDGMENT}

The author would like to thank Emeritus Professor Russell C. H. Cheng at the University of Southampton for his valuable commentaries during the writing of the draft of some theorems, and also Professor Rafael A. Díaz at the Universidad Central de Venezuela for his observations on the paper.

\section{REFERENCES}

[1] C. Audet and J. E. Dennis, Jr. Pattern search algorithms for mixed variable programming. SIAM J. Optim. 11 (2000/01), no. 3, 573-594. MR 1814033.

[2] E. Brea. Extensiones del método de Nelder Mead a problemas de variables enteras y enteras mixtas. Technical report, Universidad Central de Venezuela, 2009. Ascent work for the full professor category.

[3] C. A. Floudas. Nonlinear and mixed-integer optimization: fundamentals and applications. Oxford University Press, New York, 1995.

[4] W. E. Hart. A convergence analysis of unconstrained and bound constrained evolutionary pattern search. Evolutionary Computation 9 (2001), no. 1, 1-23.

[5] T. G. Kolda, R. M. Lewis, and V. Torczon. Optimization by direct search: new perspectives on some classical and modern methods. SIAM Rev. 45 (2003), no. 3, 385-482. MR 2046504

[6] T. G. Kolda, R. M. Lewis, and V. Torczon. Stationarity results for generating set search for linearly constrained optimization. SIAM J. Optim. 17 (2006), no. 4, 943-968 MR 2274499

[7] J. P. Lawrence. Randomized pattern search. IEEE Trans. Comput. 21 (1972), no. 4, 382-385. https://doi.org/10.1109/TC.1972.5008979

[8] J. P. Lawrence and F. P. Emad. An adaptive randomized pattern search. In Proceedings of the 1972 IEEE Conference on Decision and Control and 11th Symposium on Adaptive Processes (New Orleans, Louisiana), 421-425. The Institute of Electrical and Electronic Engineers, 1972. https://doi.org/10.1109/CDC.1972.269034

[9] K. I. M. McKinnon. Convergence of the Nelder-Mead simplex method to a nonstationary point. SIAM J. Optim. 9 (1998), no. 1, 148-158. MR 1662567

[10] R. H. Myers and D. C. Montgomery. Response Surface Methodology: Process and Product Optimization Using Designed Experiments. 2nd edition. John Wiley and Sons, New York, 2002.

[11] V. Torczon. On the convergence of pattern search algorithms. SIAM J. Optim. 7 (1997), no. 1, 1-25. MR 1430554

\section{E. Brea}

Departamento de Electrónica, Computación y Control, Escuela de Ingeniería Eléctrica, Facultad de Ingeniería, Universidad Central de Venezuela, Caracas 1053, Venezuela.

ebert.brea@ucv.ve, ebertbrea@gmail.com

Received: May 11, 2018

Accepted: March 2, 2019 